\title{
Equilibrium magnetization and magnetization relaxation of multicore magnetic nanoparticles
}

Article

Accepted Version

Ilg, P. (2017) Equilibrium magnetization and magnetization relaxation of multicore magnetic nanoparticles. Physical Review B, 95 (21). 214427. ISSN 1098-0121 doi:

https://doi.org/10.1103/PhysRevB.95.214427 Available at https://centaur.reading.ac.uk/70753/

It is advisable to refer to the publisher's version if you intend to cite from the work. See Guidance on citing.

To link to this article DOI: http://dx.doi.org/10.1103/PhysRevB.95.214427

Publisher: American Physical Society

All outputs in CentAUR are protected by Intellectual Property Rights law, including copyright law. Copyright and IPR is retained by the creators or other copyright holders. Terms and conditions for use of this material are defined in the End User Agreement.

www.reading.ac.uk/centaur 
Central Archive at the University of Reading

Reading's research outputs online 


\title{
Equilibrium magnetization and magnetization relaxation of multi-core magnetic nanoparticles
}

\author{
Patrick Ilg \\ School of Mathematical, Physical, and Computational Sciences, \\ University of Reading, Reading, RG6 6AX, United Kingdom
}

(Dated: May 25, 2017)

\begin{abstract}
Multi-core magnetic nanoparticles show promising features for biomedical applications. Their magnetic properties, however, are not well-understood to date, so that several ad hoc assumptions are often needed to interpret experimental results. Here, we present a comprehensive computer simulation study on the effect of dipolar interactions and magnetic anisotropy on the equilibrium magnetization and magnetization relaxation dynamics of monodisperse multi-core magnetic nanoparticles in viscous solvents. We include thermal fluctuations of the internal Néel relaxation via the stochastic Landau-Lifshitz-Gilbert equation coupled to rotational Brownian motion of the cluster. We find that the effective magnetic moment of the cluster is reduced compared to the non-interacting case due to frustrated dipole-dipole interactions. Furthermore, the magnetization relaxation is found to proceed in a two-step fashion with a fast initial decay being followed by a long-time relaxation. For moderate dipolar interaction strengths, the latter can be approximated quite well by an exponential decay with rate given by the sum of the relaxation rates in the immobilized state and the Brownian rotation. These findings can be helpful for a better interpretation of experimental data obtained from magnetization relaxation measurements.
\end{abstract}

PACS numbers: 75.75.-c, 75.75.Jn, 05.40.-a 


\section{INTRODUCTION}

Multi-core magnetic nanoparticles (MCMNPs) are being used more and more often in biotechnical applications such as in vitro magnetic separation and purification ${ }^{1,2}$ or for the extraction of contaminants ${ }^{3}$. Of particular relevance are also biomedical treatments ${ }^{4,5}$, such as applications in hyperthermia ${ }^{6-8}$ and as diagnostic tools, e.g. in biomedical sensing and magnetic resonance imaging ${ }^{9}$ or in magnetorelaxometry $(\mathrm{MRX})^{10-12}$. In MRX, the magnetization relaxation is measured after a strong ordering magnetic field is switched off. This technique has e.g. been used to study the binding kinetics of surface-modified MCMNPs, since the relaxation signal shows a characteristic change when Brownian rotation is suppressed due to binding ${ }^{13}$. The magnetization relaxation of magnetic nanoparticles has recently also been successfully measured in live cells ${ }^{14}$. In a similar context, MRX has also been used to show the aggregation behavior of magnetic nanoparticles in cell cultures ${ }^{15}$.

While the dynamics and magnetization relaxation of single-core magnetic nanoparticles in viscous solvents is rather well-understood ${ }^{16-19}$, much less is known for the corresponding case of multi-core particles. For single-core magnetic nanoparticles, detailed studies are available e.g. on the dependence of the Brownian relaxation time on the concentration and field strength ${ }^{20}$ or on the additional effect of internal, Néel relaxation dynamics on the magnetic relaxation behavior ${ }^{21}$. For MCMNPs, the corresponding effects are largely unknown to date which hinders further progress in their applications.

In a coordinated effort, measurements of structural and magnetic properties of single and multi-core MNPs have been compared across different laboratories ${ }^{22}$. While the hydrodynamic diameter of two types of multi-core particles could be determined quite consistently around $80 \mathrm{~nm}$ and $100 \mathrm{~nm}$, respectively, rather large differences regarding the estimated size of the individual single--core particles where found depending on the analysis techniques used. At room temperatures and in the liquid state, MCMNPs are typically found to be superparamagnetic ${ }^{9,22}$. Additional experiments on the magnetization of MCMNPs were performed ${ }^{23}$ which also revealed the effect of magnetic interactions on the effective magnetic moment of the multi-core cluster. Furthermore, the magnetization relaxation in the fluid and immobilized state has been measured in a controlled manner ${ }^{22,24}$.

The magnetization and magnetization relaxation is often analyzed in terms of the cluster moment superposition model $^{25}$. In this model, the total magnetization is assumed to result from the superposition of independent an ideal Langevin magnetization and Debye relaxation contributions according to the core-- and cluster-size distribution. Since the latter are usually unknown, log-normal size distributions are typically assumed and the parameters determined by fitting to the experimental observations ${ }^{12,25}$. Therefore, magnetization relaxation measurements are a powerful but indirect tool to infer cluster sizes and dynamics, as they are difficult to interpret or rely on questionable assumptions. For example, experimental indications for the importance of core-core interactions have been presented in Ref. 8 which are ignored in the cluster superposition model.

Computer simulation studies can be very helpful in this situation since they are able to clearly separate different interactions and effects and allow to study their individual influence on various quantities. The effective magnetization of MCMNPs has been studied by Monte-Carlo simulations where dipolar interactions and magnetic anisotropy contribute to reduce the magnetization compared to the non-interacting case ${ }^{26-28}$. It should be noted, however, that cooperativity effects that increase the effective magnetic moment have been observed in some MCMNPs ${ }^{29}$, which is thought to be caused by exchange interactions as proposed in Ref. 30. The magnetization relaxation of dry MCMNPs due to internal deterministic Landau-Lifshitz dynamics neglecting thermal fluctuations has been simulated in Ref. 31. However, we are not aware of any comprehensive simulation study on the magnetization relaxation of MCMNPs taking into account thermal fluctuations of the internal (Néel) dynamics as well as the Brownian rotation of the cluster in a viscous liquid. The present paper aims to fill this gap and provide insights into the effect of magnetic interactions on the effective magnetization and magnetization relaxation of MCMNPs.

The paper is organized as follows. The simulation model is formulated in Sect. II. Steric properties like size and shape of clusters are presented in Sect. III. Results on the effective magnetization of MCMNP in response to a static external magnetic field are shown in Sect. IV. Sect. V presents simulation results on the magnetization relaxation. Finally, some conclusions are offered in Sect. VI.

\section{MODEL FORMULATION}

We model the MCMNPs as rigid clusters formed by $N$ magnetic nanoparticles. The individual magnetic nanoparticles are assumed to be small enough so that they can be treated as magnetic mono-domain particles with magnetic moment $\mathbf{m}_{i}=m \mathbf{e}_{i}, i=1, \ldots, N$, with the three-dimensional unit vector $\mathbf{e}_{i}$ giving the magnetization direction. The magnitude of the magnetic moment is given by $m=M_{\mathrm{s}} v_{\mathrm{m}}$ with $M_{\mathrm{s}}$ the spontaneous magnetization of the magnetic material and $v_{\mathrm{m}}$ the magnetic volume of the nanoparticle. For simplicity we here consider mono-disperse samples, i.e. the magnetic moments $m$ are identical for all nanoparticles. 
The magnetization dynamics of a single magnetic nanoparticle is routinely modeled by the stochastic LandauLifshitz-Gilbert (LLG) equation ${ }^{16,21,32-34}$

$$
\frac{\mathrm{d}}{\mathrm{d} t} \mathbf{e}_{i}=-\gamma \mathbf{e}_{i} \times \mathbf{H}_{i}^{\mathrm{tot}}-\gamma \lambda \mathbf{e}_{i} \times\left(\mathbf{e}_{i} \times \mathbf{H}_{i}^{\mathrm{tot}}\right),
$$

where $\gamma(\gamma>0)$ denotes a gyromagnetic ratio and $\lambda$ a dimensionless damping parameter. The notion 'stochastic Landau-Lifshitz equation' has been used e.g. in Refs. 32 and 35 when the fluctuating field is dropped from the relaxation term. We here follow the notation of Ref. 32. The formulation of Ref. 16 follows by substituting $\gamma \rightarrow$ $\gamma /\left(1+\lambda^{2}\right)$ throughout. In the following, we choose $\lambda=0.2$ which is within the weak damping limit where both formulations become identical and which is the relevant regime for common magnetic materials ${ }^{31}$. The stochastic differential equation (1) is usually interpreted in the Stratonovich and not in Itô sense ${ }^{32}$. For the present case of constant magnitude of magnetic moments $m$, identical results are obtained for both interpretations ${ }^{36}$.

The total field acting on the magnetic moment $\mathbf{m}_{i}$ is composed of a deterministic and a fluctuating field, $\mathbf{H}_{i}^{\text {tot }}=$ $\mathbf{H}_{i}+\mathbf{H}_{i}^{\mathrm{f}}$. The deterministic field is obtained from $\mathbf{H}_{i}=-\frac{\partial \mathcal{H}}{\partial \mathbf{m}_{i}}$, where the Hamiltonian $\mathcal{H}$ contains contributions from an externally applied field $\mathbf{H}_{0}$, the anisotropy energy and dipolar interactions between nanoparticles,

$$
\begin{aligned}
\beta \mathcal{H} & =-\alpha \sum_{i=1}^{N} \mathbf{e}_{i} \cdot \mathbf{h}-\kappa \sum_{i=1}^{N}\left(\mathbf{e}_{i} \cdot \mathbf{u}_{i}\right)^{2} \\
& -Q_{\mathrm{dd}} \sum_{i<j=1}^{N} \frac{\mathbf{e}_{i} \cdot \mathbf{e}_{j}-3\left(\mathbf{e}_{i} \cdot \hat{\mathbf{r}}_{i j}\right)\left(\mathbf{e}_{j} \cdot \hat{\mathbf{r}}_{i j}\right)}{\left(r_{i j} / a\right)^{3}}
\end{aligned}
$$

where $\beta=1 /\left(k_{\mathrm{B}} T\right)$ with $k_{\mathrm{B}}$ Boltzmann's constant and $T$ the absolute temperature. The Langevin parameter is denoted by $\alpha=\beta \mu_{0} m H_{0}, H_{0}$ the magnitude of the external magnetic field and $\mathbf{h}=\mathbf{H}_{0} / H_{0}$ its direction. Next, the dimensionless strength of the anisotropy energy is given by $\kappa=\beta K v_{\mathrm{m}}$ with $K$ the anisotropy constant and $\mathbf{u}_{i}$ denoting the direction of the easy axis of nanoparticle $i$. Finally, the dimensionless strength of dipolar interactions is measured by $Q_{\mathrm{dd}}=\frac{\beta \mu_{0} m^{2}}{4 \pi a^{3}}$, with $\mu_{0}$ the permeability of free space and $a$ the radius of the individual magnetic nanoparticles. The connector vector between nanoparticle $i$ and $j$ is defined as $\mathbf{r}_{i j}=\mathbf{r}_{i}-\mathbf{r}_{j}$ with its norm $r_{i j}=\left|\mathbf{r}_{i j}\right|$ and unit vector $\hat{\mathbf{r}}_{i j}=\mathbf{r}_{i j} / r_{i j}$. All pairs of particles are included for calculating the dipolar interactions in the simulations and no truncation is applied. The fluctuating fields $\mathbf{H}_{i}^{\mathrm{f}}$ are modeled as uncorrelated, three-dimensional Gaussian random variables that obey the relations $\left\langle\mathbf{H}_{i}^{\mathrm{f}}(t)\right\rangle=0$ and $\left\langle\mathbf{H}_{i}^{\mathrm{f}}(t) \mathbf{H}_{j}^{\mathrm{f}}\left(t^{\prime}\right)\right\rangle=2 D_{\mathrm{LLG}} \delta_{i j} \delta\left(t-t^{\prime}\right) \mathbf{I}$, where $D_{\mathrm{LLG}}=\lambda /\left(1+\lambda^{2}\right)\left(k_{\mathrm{B}} T / \gamma m\right)$ and $\mathbf{I}$ denotes the three-dimensional unit matrix ${ }^{32,34}$.

Following earlier works ${ }^{26,27,31}$, we model the MCMNP as a rigid assembly of $N$ single-core magnetic nanoparticles, randomly arranged in a rather densely packed arrangement (see Sect. III). Instead of the dry case considered in Refs. 27 and 31, we here consider MCMNPs immersed in a viscous carrier liquid with viscosity $\eta$. Therefore, the MCMNPs perform rotational Brownian motion as a rigid body. While a Monte-Carlo scheme has been employed for this situation earlier to investigate the equilibrium magnetic properties ${ }^{26}$, we here aim at describing also the magnetization dynamics and therefore consider the LLG equation coupled to the rotational Brownian motion. The total torque that the external field $\mathbf{H}_{0}$ exerts is given by $\mathbf{M} \times \mathbf{H}_{0}$, where $\mathbf{M}=m \sum_{i=1}^{N} \mathbf{e}_{i}$ denotes the total magnetic moment of the MCMNP. Together with the viscous $-\zeta_{N} \boldsymbol{\omega}$ and random torque $\mathbf{R}$, the rotational Brownian motion of the easy axis $\mathbf{u}_{i}$, magnetization direction $\mathbf{e}_{i}$ and position $\mathbf{r}_{i}$ of particle $i$ is described by ${ }^{19,37}$

$$
\dot{\mathbf{u}}_{i}=\boldsymbol{\omega} \times \mathbf{u}_{i}, \quad \dot{\mathbf{e}}_{i}=\frac{\mathrm{d}}{\mathrm{d} t} \mathbf{e}_{i}+\boldsymbol{\omega} \times \mathbf{e}_{i}, \quad \dot{\mathbf{r}}_{i}=\boldsymbol{\omega} \times \mathbf{r}_{i}
$$

with the angular velocity $\boldsymbol{\omega}$ determined from the torque balance

$$
\boldsymbol{\omega}=\frac{1}{\zeta_{N}}\left[\mathbf{M} \times \mathbf{H}_{0}+\mathbf{R}\right]
$$

where $\mathbf{R}$ denotes a three-dimensional Gaussian white noise. While the stochastic LLG equation (1) gives the time derivative of the magnetization direction $\frac{\mathrm{d}}{\mathrm{d} t} \mathbf{e}_{i}$ in quiescent conditions, Eq. (3) accounts for the overall rotation of the MCMNP due to overdamped Brownian motion in a carrier liquid. For immobile MCMNP, $\boldsymbol{\omega}=0$ and $\dot{\mathbf{e}}_{i}=\frac{\mathrm{d}}{\mathrm{d} t} \mathbf{e}_{i}$. It is worth to point out that Eqs. (3), (4) can also be derived by generalizing the so-called "egg-model" of Ref. 38 to rigid clusters (see appendix A). Since the system is translationally invariant, we consider only rotations around the center of mass.

The equations of motion (1) and (3), (4) define the magnetization dynamics of the MCMNP system. The numerical algorithm implementing the equations of motion is described in Appendix B. The equations of motion employed 
here apply to homogeneously magnetized (single-domain) nanoparticles that can be modeled as a point dipole at their centers ${ }^{17,19}$. Nanoparticle assemblies in multi-core particles are often densely packed and coated with a shell of e.g. starch $^{23}$ or silica ${ }^{9}$ or carboxymethyldextran ${ }^{24}$, to mention a few. Therefore, we assume the nanoparticles are immobilized concerning relative translation and rotation with respect to each other, so that only internal Néel relaxation and rigid Brownian rotation of the multi-core particle are present. These assumptions have also been used e.g. in Ref. 27.

As will be shown in Sect. III, the MCMNPs considered in this work are to a very good approximation dense and spherical. Therefore we use the rotational friction coefficient $\zeta_{N}=8 \pi \eta R_{\mathrm{h}}^{3}$ of a sphere, where $R_{\mathrm{h}}$ denotes the hydrodynamic radius of the MCMNP. The noise in Eq. (3) is specified by $\langle\mathbf{R}(t)\rangle=0$ and $\left\langle\mathbf{R}(t) \mathbf{R}\left(t^{\prime}\right)\right\rangle=\sqrt{2 k_{\mathrm{B}} T \zeta_{N}} \delta(t-$ $\left.t^{\prime}\right) \mathbf{I}$. The stochastic differential equation for the Brownian rotation (3) should be interpreted in the Stratonovich sense $^{34}$.

Note that Eq. (3) corresponds to the so-called overdamped regime where inertia effects can be neglected. Since the inertia time scale $\tau_{I}=I / \zeta_{N}$, with $I$ the moment of inertia of the MCMNP, is typically several order of magnitude smaller than the Brownian relaxation time $\tau_{\mathrm{B}}=\beta \zeta_{N} / 2$, Brownian motion is considered an excellent approximation for the dynamics of colloids ${ }^{19,37}$.

For the LLG equation (1), $\tau_{0}=m /\left[2 \lambda \gamma k_{\mathrm{B}} T\right]$ gives the characteristic diffusion time of the magnetic moment orientations within a nanoparticle when anisotropy energy is negligible ${ }^{16,32}$. While $\tau_{0}$ is often short, typically on the order of $10^{-10} \mathrm{~s}$, the corresponding relaxation time $\tau_{\mathrm{N}}$ is steeply increasing with the dimensionless anisotropy parameter $\kappa$. For $\kappa \gtrsim 3$, Brown derived the approximate relation $\tau_{N} \approx \sqrt{\pi} \tau_{0} e^{\kappa} /\left(2 \kappa^{3 / 2}\right)^{16}$. For the present range of parameters, we find that $\tau_{\mathrm{N}} \approx \tau_{0} \exp [\kappa / 2]$ provides a satisfactory description for $\kappa \lesssim 4$ (see Sect. V).

It is appropriate to mention that the Néel relaxation time $\tau_{\mathrm{N}}$ is independent of the cluster size, whereas the Brownian relaxation increases as $\tau_{\mathrm{B}} \sim R_{\mathrm{h}}^{3}$. For clusters with $\tau_{\mathrm{N}} \ll \tau_{\mathrm{B}}$, the proposed scheme might become inefficient if very long simulations are needed since the fast Néel as well as the long time Brownian rotation needs to be covered. A possible remedy for this situation in the case of single nanoparticle has been proposed ${ }^{39}$. Note, however, that for magnetization relaxation, the present scheme remains adequate also for $\tau_{\mathrm{N}} \ll \tau_{\mathrm{B}}$ as the faster process will dominate the relaxation.

For iron oxide nanoparticles that are most often used in biomedical applications, with anisotropy constant $K=$ $10^{4} \mathrm{~J} / \mathrm{m}^{3}$, spontaneous magnetization $M_{\mathrm{s}}=370 \mathrm{kA} / \mathrm{m}$, and for typical values of the magnetic core $a_{\mathrm{c}}=4 \mathrm{~nm}$ (with magnetic volume $v_{\mathrm{m}}=\frac{4}{3} \pi a_{\mathrm{c}}^{3}$ ) and $a=5 \mathrm{~nm}^{15}$, we find values of the dimensionless anisotropy constant of $\kappa \approx 0.6$ and dipolar interaction parameter $Q_{\mathrm{dd}} \approx 1.9$, whereas for $a_{\mathrm{c}}=6 \mathrm{~nm}$ and $a=7 \mathrm{~nm}$ we find $\kappa \approx 2.2$ and $Q_{\mathrm{dd}} \approx 7.9$. In the following, we use comparable values of these interaction parameters. Note that the more commonly used dipolar interaction parameter $Q_{\mathrm{dd}}^{*}=\beta \mu_{0} m^{2} /\left(4 \pi \sigma^{3}\right)=Q_{\mathrm{dd}}(a / \sigma)^{3}$ is based on the steric distance $\sigma=2 a$ between two nanoparticles ${ }^{19}$ and therefore $Q_{\mathrm{dd}}^{*}=Q_{\mathrm{dd}} / 8$. The MCMNP investigated in Ref. 23 (BNF starch), the Brownian relaxation of a cluster of $N=100$ particles was estimated as $\tau_{\mathrm{B}} \approx 3.5 \times 10^{-4} \mathrm{~s}$. Depending on the size of the individual nanoparticles $\left(a_{\mathrm{c}}=5 \ldots 10 \mathrm{~nm}\right)$, the Néel time ranges between $\tau_{\mathrm{N}} \approx 10^{-5} \ldots 10^{-1} \mathrm{~S}$

\section{CLUSTER PREPARATION AND STERIC PROPERTIES}

We consider a MCMNP as an assembly of $N$ single-core magnetic nanoparticles that form a rigid cluster. Since we do not attempt to model the actual synthesis that leads to the formation of MCMNPs used in experiments, there is some ambiguity in building the clusters in simulations. Here, two different methods are considered in Sects. III A and III B, respectively, and their structural properties are compared in Sect. III C.

\section{A. Dense random clusters}

We first follow earlier works ${ }^{27}$ and build a cluster of $N$ particles successively by adding a single spherical particle to the surface of an already existing, randomly chosen particle. If after 100 trials no configuration without overlaps is achieved, another particle is selected at random. The procedure is repeated until the cluster contains $N$ particles at positions $\mathbf{r}_{i}, i=1, \ldots, N$. Then, a random unit vector $\mathbf{u}_{i}$ is associated with each of the $N$ particles denoting the orientation of its easy axis. The algorithm is described in more detail in Ref. 27. One realization of such a cluster is shown in Fig. 1. 


\section{B. Clusters resulting from diffusion-limited colloidal aggregation}

While the algorithm described in Sect. III A is somewhat artificial, a slightly more realistic model to build a cluster might be obtained by using the concept of diffusion-limited colloidal aggregation (DLCA) ${ }^{40}$. Also in DLCA, the cluster is build sequentially by starting with an initial seed and adding one particle at a time. However, contrary to random trial attachments, the Brownian dynamics of a particle attracted to the cluster is solved until it attaches to a particle of the cluster when the distance becomes smaller than $2 a$. In the present case, the new particle is initialized with random position and random orientation of its magnetic moment at a distance sufficiently far away from the initial seed. Then the translational and rotational Brownian motion of the particle is integrated with a first-order Euler-Maruyama scheme with time step $\Delta t=10^{-2} \tau_{\mathrm{B}, 1}$, taking into account dipolar as well as LennardJones interactions, $\beta \Phi\left(r_{i j}\right)=4 \varepsilon\left[\left(\sigma / r_{i j}\right)^{12}-\left(\sigma / r_{i j}\right)^{6}\right]$, where $\varepsilon$ is the dimensionless interaction strength ${ }^{19}$. We here use $\sigma=a$ instead of the usual $\sigma=2 a$ to arrive at denser clusters. In addition, we apply a weak radial drag force of strength $0.1 k_{\mathrm{B}} T / a$ directed towards the initial seed particle. The resulting particle drift is known to lead to effectively uniform and dense clusters ${ }^{41}$. In the following we set the dimensionless Lennard--Jones interaction parameter $\varepsilon=4$ unless explicitly stated otherwise.
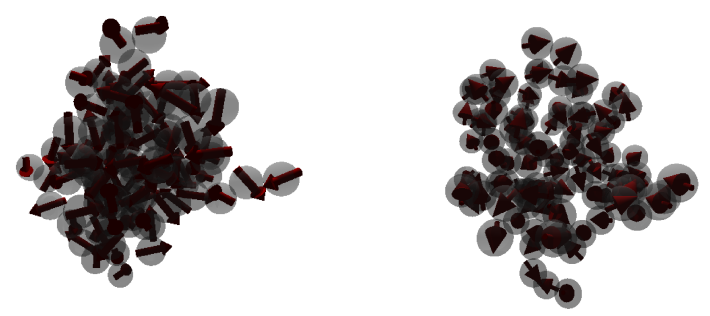

FIG. 1. Left: Visualization of a dense random cluster containing $N=100$ nanoparticles prepared as described in Sect. III A. Right: Visualization of a cluster containing $N=100$ nanoparticles prepared by DLCA with $Q_{\mathrm{dd}}=2$ and $\varepsilon=4$ as described in Sect. III B.

\section{Size and shape of clusters}

We characterize the resulting shape of the MCMNP by different quantities: (i) the radius of gyration $R_{\mathrm{g}}$, (ii) the hydrodynamic radius $R_{\mathrm{h}}$, (iii) the asphericity $A$, and (iv) the prolateness $S$. The radius of gyration is a frequently used measure for the size of a cluster ${ }^{42}$

$$
R_{\mathrm{g}}^{2}=\frac{1}{N} \sum_{i=1}^{N}\left(\mathbf{r}_{i}-\mathbf{r}_{\mathrm{cm}}\right)^{2}+a^{2}
$$

where $\mathbf{r}_{\mathrm{cm}}$ denotes the center of mass of the cluster and $a$ the radius of an individual nanoparticle $\left(\right.$ with $4 \pi a^{3} / 3=v_{\mathrm{m}}$ if the steric shell of the nanoparticle can be neglected). The additional term $a^{2}$ is included in Eq. (5) to ensure the correct limit of $R_{\mathrm{g}}$ for small cluster sizes. Concerning Brownian motion of the MCMNP, the hydrodynamic radius $R_{\mathrm{h}}$ of the cluster is more relevant than $R_{\mathrm{g}}$. We here use a rather simple formula for $R_{\mathrm{h}}$ due to Kirkwood and Riseman ${ }^{43}$,

$$
\frac{N a}{R_{\mathrm{h}}}=1+\frac{1}{N} \sum_{i \neq j} \frac{a}{\left|\mathbf{r}_{i}-\mathbf{r}_{j}\right|}
$$

which provides quite accurate results for colloidal aggregates ${ }^{42}$.

Deviations from a spherical shape can be quantified by the asphericity $A=3 \operatorname{Tr}\left(\hat{\mathbf{Q}}^{2}\right) /\left[2 R_{\mathrm{g}}^{4}\right]$ and the prolateness $S=27 \operatorname{det}(\hat{\mathbf{Q}}) / R_{\mathrm{g}}^{6}$, where $\mathbf{Q}=\frac{1}{3} a^{2} \mathbf{I}+\frac{1}{N} \sum_{i=1}^{N}\left(\mathbf{r}_{i}-\mathbf{r}_{\mathrm{cm}}\right)\left(\mathbf{r}_{i}-\mathbf{r}_{\mathrm{cm}}\right)$ is the gyration tensor, $R_{\mathrm{g}}^{2}=\operatorname{Tr}(\mathbf{Q})$, and $\hat{\mathbf{Q}}=$ $\mathbf{Q}-\left(R_{\mathrm{g}}^{2} / 3\right) \mathbf{I}^{44}$. Both quantities $A$ and $S$ are dimensionless. The asphericity obeys $0 \leq A \leq 1$ where $A=0$ holds only for a spherical configuration. The prolateness $S$ is bounded by $-1 / 4 \leq S \leq 2$, is positive for prolate, negative for oblate and vanishes for spherical configurations.

Figure 2 shows the gyration and hydrodynamic radius, $R_{\mathrm{g}}$ and $R_{\mathrm{h}}$, in units of the particle radius $a$ as a function of the number of nanoparticles $N$ in the cluster. The results shown in the following are averaged over 50 statistically 
independent realizations of the clusters and error bars correspond to one standard deviation of the independent samples. Both, $R_{\mathrm{g}}$ and $R_{\mathrm{h}}$ give very similar values with $R_{\mathrm{h}} \sim N^{\nu}$ with exponent $\nu \approx 0.32$ and $\nu \approx 0.38$ for random dense and DLCA clusters, respectively. These values for $\nu$ corresponds to dense clusters and confirm and quantify the conclusion reached in Ref. 27 for clusters prepared randomly as described in Sect. III A. Moreover, the inset in Fig. 2 shows that both, the asphericity $A$ and the prolateness $S$ are small, which indicates that the clusters can be considered as spherical to a good approximation, at least for $N \gtrsim 50$. Also experiments found rather dense and nearly spherical clusters in the range $N \approx 50 \ldots 300$ using different synthesis routes ${ }^{8,9,24}$. We emphasize again that in the interest of better understanding and to facilitate the analysis, we limit our study to the strict monodisperse case where all individual nanoparticles have the same size and all clusters are composed of the same number $N$ of nanoparticles. Due to the random nature of their preparation, the clusters do show a small distribution of sizes. For example, clusters of $N=100$ particles show an average hydrodynamic radius of $\left\langle R_{\mathrm{h}}\right\rangle \approx 5.65$ with a standard deviation of 0.13 for random dense clusters and $\left\langle R_{\mathrm{h}}\right\rangle \approx 6.27$ and standard deviation 0.20 for DLCA clusters.

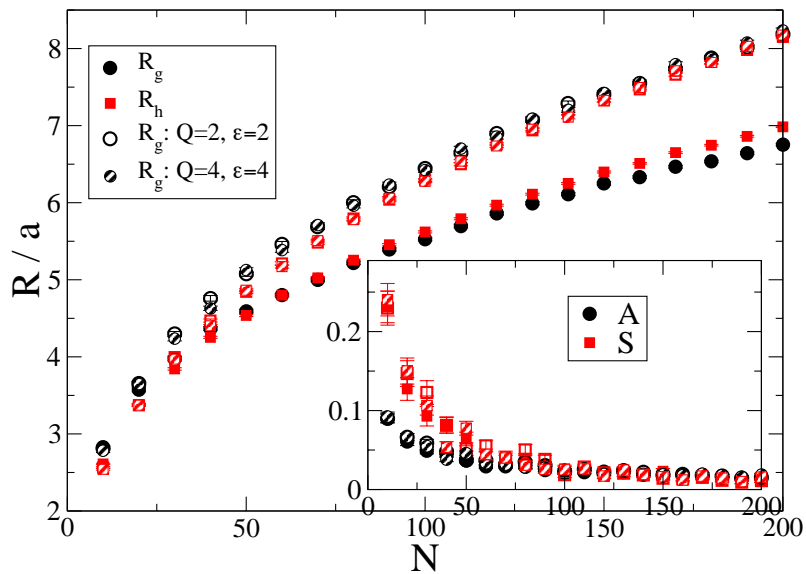

FIG. 2. Main panel: Circles and square show the radius of gyration $R_{\mathrm{g}}$, Eq. (5), and hydrodynamic radius $R_{\mathrm{h}}$, Eq. (6), as a function of the number $N$ of nanoparticles in the cluster. Filled symbols correspond to dense random clusters (Sect. III A), whereas open and shaded symbols show results for DLCA clusters (Sect. IIIB) for $Q_{\mathrm{dd}}=2, \varepsilon=2$ and $Q_{\mathrm{dd}}=4, \varepsilon=4$, respectively. The inset shows the asphericity $A$ and prolateness $S$ of the clusters as a function of $N$.

\section{EFFECTIVE MAGNETIZATION}

In the algorithm proposed in Refs. 23 and 27 and briefly described in Sect. III A for the preparation of random dense clusters, the magnetic moments of the individual nanoparticles, $\mathbf{m}_{i}=m \mathbf{e}_{i}, i=1, \ldots, N$ are chosen independent and randomly from an isotropic distribution. Therefore, by construction the mean-squared total magnetic moment $\left\langle\mathbf{M}^{2}\right\rangle$ shows a perfect random walk scaling, $\left\langle\mathbf{M}^{2}\right\rangle=N m^{2}$ (see Ref. 37). This is not necessarily the case for clusters formed via DLCA, since the magnetic moments are assigned initially and dynamically evolve during the Brownian dynamics evolution until they freeze when hitting the cluster. Nevertheless, from the simulations we find that also in the case of DLCA, the random walk scaling of the mean-squared magnetization provides a very good approximation, see Fig. 3.

We now consider the average magnetic moment of a multi-core particle, $\langle\mathbf{M}\rangle=m\left\langle\sum_{i=1}^{N} \mathbf{e}_{i}\right\rangle$, induced by an external magnetic field $\mathbf{H}_{0}$. We numerically solve the equations of motion Eqs. (3), (4) subject to torques resulting from (2) as described in Appendix B. These simulations are run for $1000 \tau_{0}$ and repeated for 50 independent realizations of dense random clusters and DLCA clusters prepared as described in Sect. III. Figure 4 shows the resulting magnetization $M=\mathbf{M} \cdot \mathbf{H}_{0} / H_{0}$ as a function of the Langevin parameter $\alpha=\mu_{0} m H_{0} / k_{\mathrm{B}} T$. We observe the typical superparamagnetic behavior well-known for colloidal magnetic fluids ${ }^{17,19}$ : a linear increase of $\langle\mathbf{M}\rangle$ for weak external fields $\alpha$ and saturation for very strong fields. Contrary to the case of interacting individual magnetic nanoparticles that are free to move relative to each other, increasing dipolar interaction strength within the cluster lead to a reduction of the resulting magnetization. The reduced magnetization of multi-core particles due to frustrated dipole-dipole interaction has been found already in Ref. ${ }^{26}$. Our results confirm their finding and provide a check of the implementation of our algorithm 


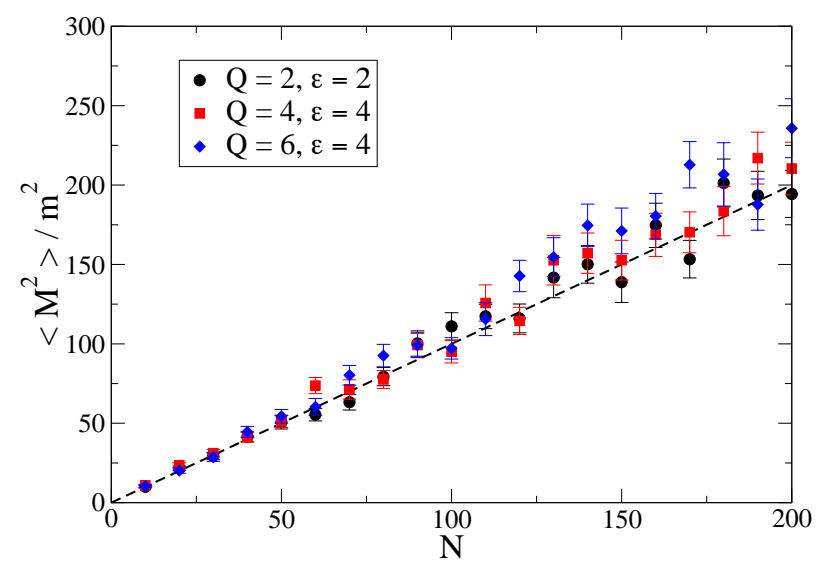

FIG. 3. Mean-squared magnetization $\left\langle\mathbf{M}^{2}\right\rangle$ in units of $m^{2}$ as a function of the number $N$ of nanoparticles in the cluster. The clusters were generated by DLCA for different values of dipolar interaction strengths $Q_{\mathrm{dd}}$ and Lennard-Jones interaction $\varepsilon$. The dashed line indicates the random walk scaling $\left\langle\mathbf{M}^{2}\right\rangle=N m^{2}$.

where rotational Brownian motion of the cluster as a whole is also present. Figure 4 shows a second important result: the resulting magnetization of dense random clusters and DLCA clusters are almost identical for the parameters investigated. This insensitivity to details of the cluster shape is reassuring for applications since cluster shapes are often not well known experimentally.

Experimentally measured magnetization curves are routinely analyzed in terms of superpositions of Langevin functions which are meant to represent polydispersity effects ${ }^{45}$. Since we deliberately disregard polydispersity in the present study, can the resulting magnetization of the cluster as a whole be faithfully described by the Langevin function, i.e. a single effective magnetic dipole moment $m_{\mathrm{eff}}$ ? In order to address this question, we first fix the known saturation magnetic moment $M_{\text {sat }}=N m$. Then, we compare the simulation data for the magnetization curve $M\left(H_{0}\right)$ with $M=M_{\text {sat }} L\left(m_{\text {eff }} H_{0} / k_{\mathrm{B}} T\right)$, where $L(x)=\operatorname{coth}(x)-1 / x$ denotes the Langevin function. The solid line in Fig. 4 shows the Langevin magnetization for $m_{\text {eff }}=m$, i.e. for an ensemble of $N$ non-interacting nanoparticles. For moderate dipolar interaction strengths $Q_{\mathrm{dd}} \lesssim 4$, very good agreement between simulation data and the Langevin curve is observed. For increasing dipolar interaction strength, we observe that the magnetization is reduced compared to the Langevin function, in agreement with earlier simulation results ${ }^{26}$. Overall, for the present range of parameters (with $\left.Q_{\mathrm{dd}} \lesssim 8\right)$, we find that the equilibrium magnetization of the MCMNP is to a good approximation given by Langevin magnetization with effective dipole moment $m_{\mathrm{eff}} \approx m$.

\section{MAGNETIZATION RELAXATION}

In order to simulate the magnetization relaxation from a well-defined initial condition, a strong external magnetic field $\mathbf{H}_{0}$ with Langevin parameter $\alpha=20$ is applied for a time interval $t_{0}=10 \tau_{0}$ so that dipole moments become strongly aligned in field direction. At $t=t_{0}$, the external field is instantaneously switched off and the decay of the magnetization $\langle M\rangle(t)$ is monitored. The simulations are repeated for 900 (in same cases 2000 or 4000 ) statistically independent clusters.

Below, we focus on two cases, $\tau_{\mathrm{B}} \approx \tau_{\mathrm{N}}$ and $\tau_{\mathrm{B}} \gg \tau_{\mathrm{N}}$. First, we set the Brownian relaxation time of a single nanoparticle as $\tau_{\mathrm{B}, 1}=\tau_{0}$. The effective Brownian relaxation time of a cluster with hydrodynamic radius $R_{\mathrm{h}}$ is $\tau_{\mathrm{B}}=\tau_{\mathrm{B}, 1}\left(R_{\mathrm{h}} / a\right)^{3}$. For a cluster containing $N=100$ nanoparticles, we find $\tau_{\mathrm{B}} \approx 180 \tau_{\mathrm{B}, 1}$ and $\tau_{\mathrm{B}} \approx 250 \tau_{\mathrm{B}, 1}$, for random dense and DLCA clusters, respectively. Results for $Q_{\mathrm{dd}}=2$ and different values of the magnetic anisotropy parameter $\kappa$ are shown in Fig. 5 for $\tau_{\mathrm{B}, 1}=\tau_{0}$, i.e. $\tau_{\mathrm{B}} \gg \tau_{\mathrm{N}}$. First of all note that again the results for random dense clusters and DLCA clusters in most cases are indistinguishable within statistical uncertainties. The insensitivity of the magnetization relaxation to the details of the cluster structure is an important result especially in view of applications in MRX.

The magnetization relaxation shown in Fig. 5 can be thought to be composed of two steps. First, after the external field has been switched off, a fast initial decay due to the fast alignment of the particles' magnetic moments with their 


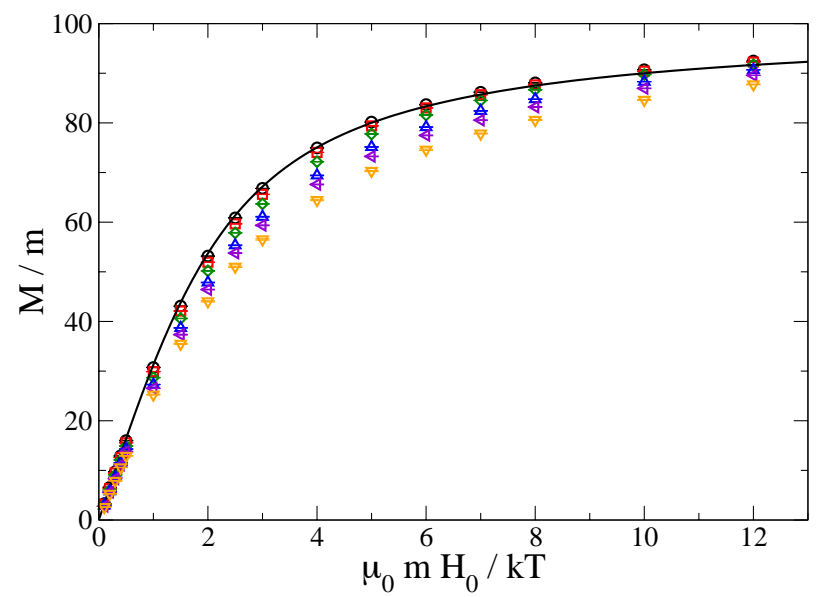

FIG. 4. Magnetization of clusters of $N=100$ magnetic nanoparticles as function of normalized applied field. The dimensionless dipolar interaction strength increases from top to bottom as $Q_{\mathrm{dd}}=2,4,8,16,24,40$. The damping and anisotropy parameter are chosen as $\lambda=0.2, \kappa=1$, respectively. Results for random dense clusters and DLCA clusters are indistinguishable on this scale. The solid line shows the Langevin magnetization.

individual easy axes since the misalignment is no longer compensated for by the energy due to the external magnetic field. We corroborate this interpretation by the observation that the magnetic anisotropy energy $-\kappa \sum_{i}\left(\mathbf{e}_{i} \cdot \mathbf{u}_{i}\right)^{2}$ steeply decreases at the same time (not shown). In a second regime, after $t \approx t_{0}+\tau_{0}$, the magnetization relaxation changes slope as relaxation now occurs mainly due to Néel relaxation of the individual particles. For the present choice of $\tau_{\mathrm{B}}$, the orientation of the clusters changes only weakly on the timescale of the relaxation. For $\kappa \gtrsim 2$, short- and long-time relaxation are separated by a small under- and overshoot. We note that in both regimes the magnetization decays exponentially to a good approximation.

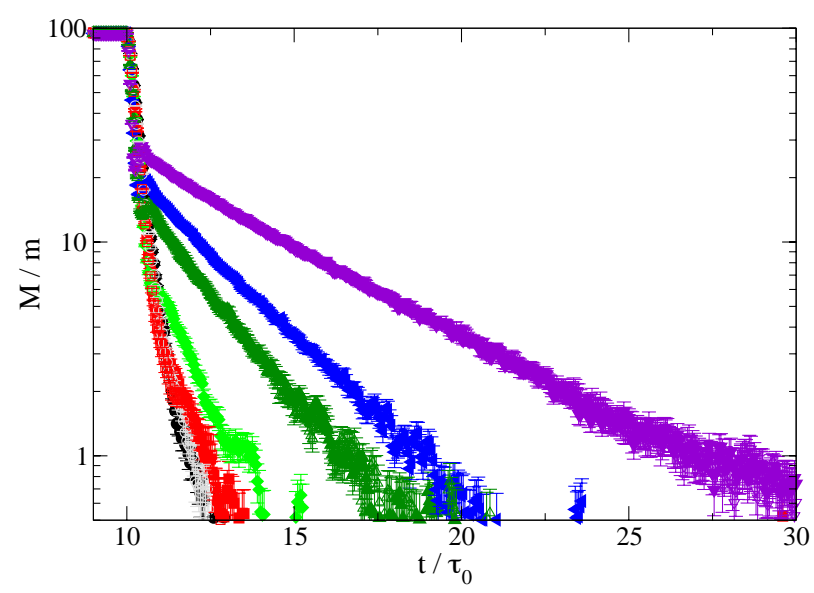

FIG. 5. Magnetization relaxation of DLCA clusters of $N=100$ nanoparticles after switching off a strong field at $t=10 t_{0}$. The normalized magnetization is shown as a function of dimensionless time $t / \tau_{0}$ in a semi-logarithmic plot. From bottom to top, the magnetic anisotropy parameter increases as $\kappa=0.5,1,1.5,2.5,3,4$. Other parameters are chosen as $\tau_{\mathrm{B}, 1}=\tau_{0}\left(\tau_{\mathrm{B}} \gg\right.$ $\left.\tau_{\mathrm{N}}\right), Q_{\mathrm{dd}}=2, \lambda=0.2$. Closed and open symbols indicate results for dense random clusters and DLCA clusters.

When clusters of magnetic nanoparticles are suspended in a viscous medium, the rotational Brownian motion of the cluster interferes with the internal magnetization relaxation. Even when reducing the Brownian relaxation time 
$\tau_{\mathrm{B}}$ considerably, we still observe the characteristic two-step relaxation (see Fig. 6). The initial relaxation happens so fast that it is only indirectly affected by the additional Brownian motion of the cluster. In fact, the initial relaxation is less pronounced when Brownian rotation is significant since individual magnetic moments are better aligned with their easy axes compared with the immobilized case. The final relaxation, however, is strongly affected by Brownian motion which leads to a significantly faster decay of the magnetization. This important effect makes MRX such a powerful technique.

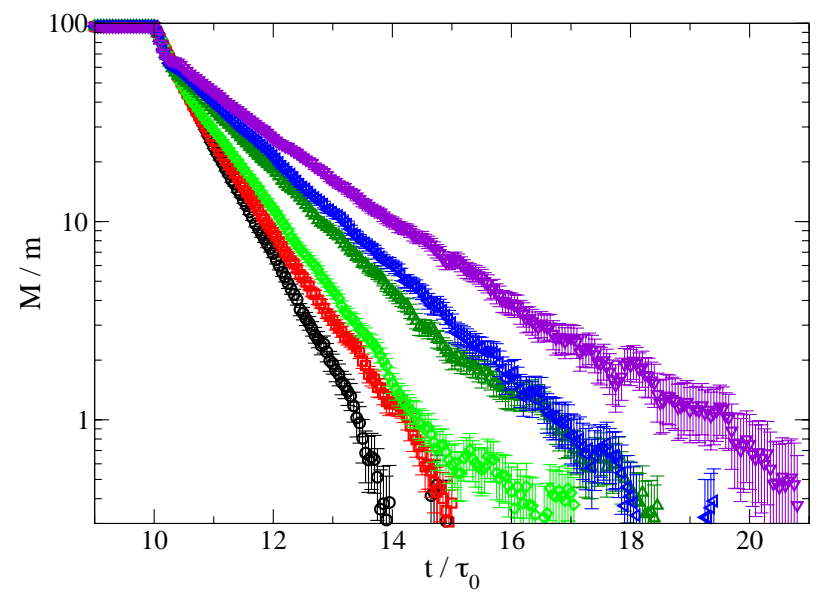

FIG. 6. Same as Fig. 5 but for $\tau_{\mathrm{B}, 1}=0.01 \tau_{0}\left(\tau_{\mathrm{B}} \approx \tau_{\mathrm{N}}\right)$. Only results for DLCA clusters are shown.

Next, we investigate the influence of dipolar interactions on the magnetization relaxation. Figure 7 a) and b) show the plots corresponding to Figs. 5 and 6 , respectively, but for a fixed value of the magnetic anisotropy $\kappa$ and varying the dipolar interaction strength $Q_{\mathrm{dd}}$. For slow Brownian relaxation, we find from Fig. 7 a) that stronger dipolar interactions lead to a more pronounced initial decay of the magnetization, with little effect on the long-time relaxation. In the case of comparable Néel and Brownian relaxation, Fig. 7 b), dipolar interactions have even less effect on the long-time relaxation.

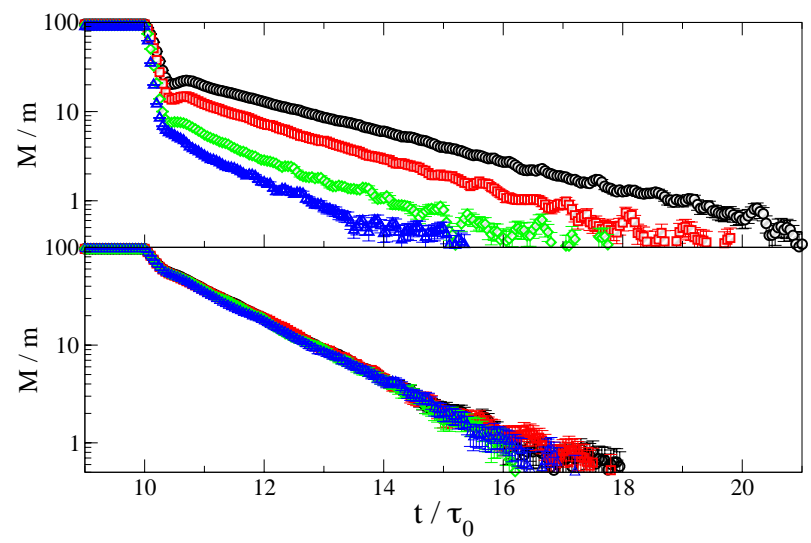

FIG. 7. Panel a) and b) show the same as Figs. 5 and 6 but for $\kappa=2.5$. Circles, squares, diamonds and triangles denote the results for $Q_{\mathrm{dd}}=1,2,4,6$, respectively. Only results for DLCA clusters are shown. 
It should be added that the simple behaviour just described does not hold for strong dipolar interactions. In fact, we observe for the case $\tau_{\mathrm{B}} \approx \tau_{\mathrm{N}}$ that the magnetization relaxation slows down considerably for increasing dipolar interaction strength above $Q_{\mathrm{dd}} \approx 16$, see Fig. 8. In this regime, the relaxation is slower than exponential. For $Q_{\mathrm{dd}}=40, \kappa=2.5$, for example, we find that the long-time relaxation is approximately described by a power-law decay $M(t) \sim t^{-\delta}$ with $\delta \approx 1.23 \pm 0.01$. These findings are in qualitative agreement with earlier studies that found slow magnetic relaxation in two-dimensional nanoparticle assemblies due to the combined effect of thermal activation due to the anisotropy energy barrier combined with dipolar interactions ${ }^{35,46}$. Even though those works considered regular nanoparticle arrays that are furthermore not performing rotational Brownian motion, the underlying mechanism leading to slow relaxation seems to be relevant also for the present case.

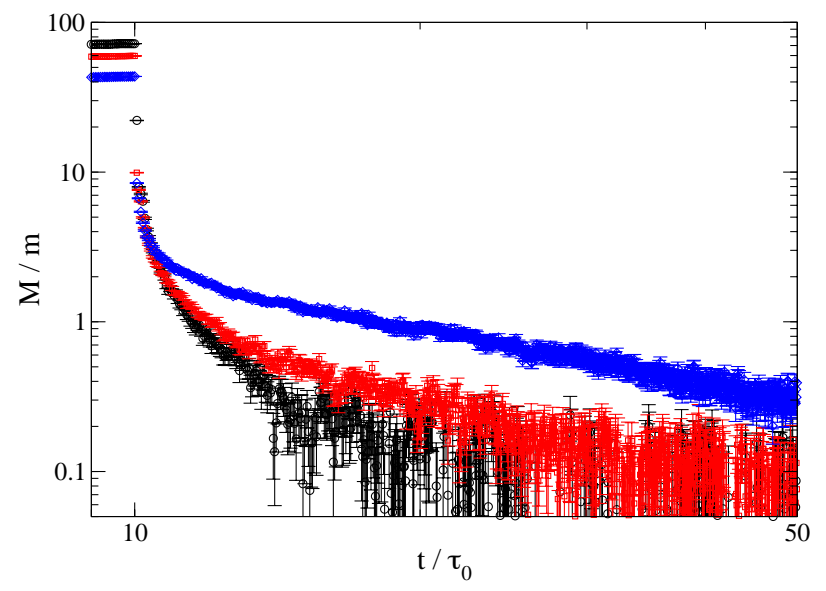

FIG. 8. Same as panel a) in Fig. 7 in a double-logarithmic plot. Only results for DLCA clusters with $Q_{\text {dd }}=40,24,16$ (from top to bottom at the right) are shown. To improve statistics, averages are taken over 4000 realizations of clusters each consisting of $N=100$ particles.

Since the long-time relaxation of the magnetization is to a good approximation exponential (at least for not too strong dipolar interactions), we define an effective magnetization relaxation time $\tau_{M}$ from $M(t) \sim \exp \left[-t / \tau_{M}\right]$ for times $t>t_{0}+\tau_{N}$, where $t_{0}$ denotes the time when the external magnetic field is switched off. Figure 9 shows the results for the effective relaxation times $\tau_{M}$ as a function of the anisotropy constant $\kappa$. In the case that Brownian rotation is much slower than Néel relaxation, we find that the effective magnetization relaxation time of the cluster follows the Néel relaxation time of an individual nanoparticle with $\bar{\tau}_{M} \approx 0.75 \tau_{\mathrm{N}}$ as a rough, empirical approximation (see dashed line in Fig. 9). That $\bar{\tau}_{M}$ is smaller than $\tau_{\mathrm{N}}$ is probably related to the frustrated dipole-dipole interactions that help to mediate the reorientation process. When the timescale of rotational Brownian motion becomes comparable to the Néel relaxation time, the effective magnetization relaxation time is significantly reduced. In the presence of Néel and Brownian relaxation, we define in analogy to the case of single magnetic nanoparticles an effective relaxation time ${ }^{38}$ $\tau_{M}$ by

$$
\frac{1}{\tau_{M}}=\frac{1}{\bar{\tau}_{M}}+\frac{1}{\tau_{\mathrm{B}}}
$$

where $\tau_{\mathrm{B}}$ is the Brownian relaxation time of the cluster and $\bar{\tau}_{M} \approx 0.75 \tau_{\mathrm{N}}$ the effective magentisation relaxation time when Brownian rotation is blocked. The dash-dotted line in Fig. 9 shows that Eq. (7) provides a good description of $\tau_{M}$ obtained from the simulation data. Therefore, we can conclude that the magnetization relaxation of a rigid cluster proceeds via Brownian rotation and Néel relaxation with their respective relaxation rates adding up as in the single nanoparticle case. 


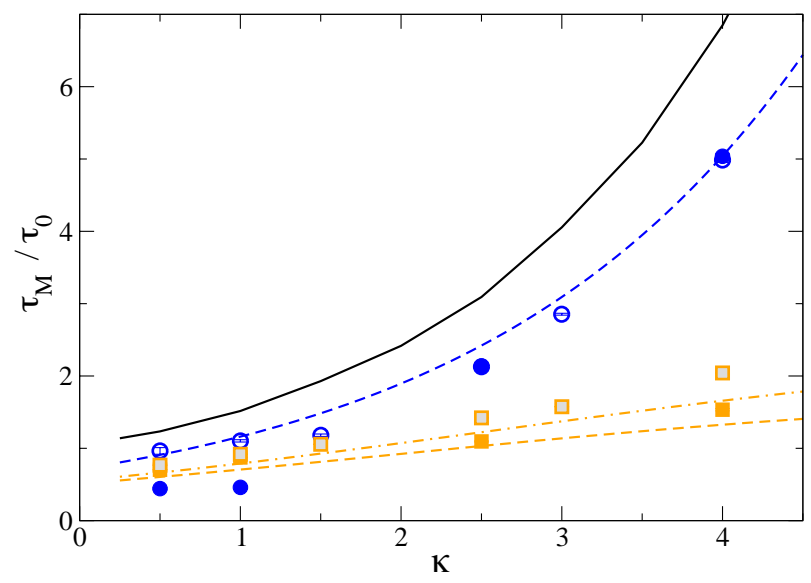

FIG. 9. Effective long-time magnetization relaxation time $\tau_{M}$ as a function of the dimensionless anisotropy constant $\kappa$. Circles and squares correspond to $\tau_{\mathrm{B}, 1}=\tau_{0}$ and $\tau_{\mathrm{B}, 1}=0.01 \tau_{0}$, respectively. Filled and open symbols to random dense and DLCA clusters, respectively. The dimensionless dipolar interaction and damping parameter are chosen as $Q_{\mathrm{dd}}=2, \lambda=0.2$, respectively. The solid line indicates the result for the effective Néel relaxation $\tau_{\mathrm{N}}$ of individual magnetic nanoparticles with random but frozen orientation of their easy axes, see Appendix C. Dashed line is the empirical relation $\tau_{M}=0.75 \tau_{\mathrm{N}}$. Finally, the dash-dotted line indicates the effective relaxation time $\tau_{M}$ defined by Eq. (7) when Néel and Brownian relaxation are present at the same time.

\section{CONCLUSIONS}

We present here a computer simulation study on the static and dynamic magnetization behavior of multi-core magnetic nanoparticles. The internal magnetization dynamics of the nanoparticles is modeled by the stochastic Landau-Lifshitz-Gilbert equation (1), where we also take dipolar interactions into account. The overall rotation of the cluster in a viscous liquid is modeled by rotational Brownian motion. We generate clusters of nanoparticles in a step-by-step manner, either by randomly attaching to previous particles as in ${ }^{26,27}$ or via DLCA. Once formed, the clusters are assumed to remain rigid. As a first step and to simplify the interpretation of our results, we here consider only the strictly monodisperse case where all individual magnetic nanoparticles are of the same size and all clusters contain the same number of nanoparticles.

Although clusters formed by DLCA are somewhat more loosely packed than those formed by random attachments, in both cases the clusters can be considered to be rather dense and roughly spherical. From equilibrium simulations, we determine the effective magnetization of the clusters. Due to the frustration of dipolar interactions, the effective magnetization of the cluster is reduced compared to the non-interacting case ${ }^{23,26}$. Therefore, the effective magnetization is a decreasing function of the dipolar interaction strength $Q_{\mathrm{dd}}$. For the parameter range investigated here, the effective magnetization can nevertheless be described rather well by the Langevin magnetization. It is reassuring to note that details of cluster structure seem to be irrelevant in this case, as almost identical results are obtained for both type of clusters considered.

Turning to the relaxation of the effective magnetization following the switching off of a strong external field, we observe a characteristic two-step scenario. A fast initial relaxation where magnetic moments align with their individual easy axes is followed by a slower long-time relaxation towards vanishing net magnetization. The long-time relaxation is to a good approximation exponential, allowing us to reliably define an effective magnetization relaxation time $\tau_{M}$ of the cluster. When Brownian motion is much slower than the internal magnetization dynamics, $\tau_{\mathrm{B}} \gg \tau_{\mathrm{N}}$, we find that $\tau_{M}$ roughly follows the behavior of the Néel relaxation of single magnetic nanoparticles since the internal Néel relaxation is the dominant contribution in this case. In the regime where Brownian and Néel relaxation compete, $\tau_{\mathrm{B}} \approx \tau_{\mathrm{N}}$, we find that both relaxation processes contribute to the magnetization relaxation leading to an effective relaxation rate, Eq. (7), in analogy to the case of single magnetic nanoparticles. It is interesting to note that magnetic anisotropy strongly affects magnetization relaxation whereas dipolar interactions seem to play a less dominant role in the parameter range investigated here. Also for the case of magnetization relaxation, we find that details of the cluster shape are rather irrelevant, except maybe at high values of the dimensionless anisotropy constant $\kappa$. A word of caution is in order here as slow, non-exponential relaxation is found for the case of strong dipolar interactions. 
With the present study, we provide some steps towards better interpreting magnetization measurements on MCMNPs. In particular, our study justifies the use of an effective single particle model of the cluster with effective magnetic moment and relaxation time. The relative insensitivity of the results to details of the cluster structure are very reassuring. For a more quantitative comparison to experimental results, the simulations need to be extended to include polydispersity in the nanoparticle size as well as in the number $N$ of nanoparticles within the cluster. Preliminary results indicate that moderate levels of polydispersity in $N$ do not significantly affect the present results. In addition, cooperativity effects that lead to an increase of the effective magnetic moment of the cluster might need to be included for specific cases. With these extensions, simulations of the kind performed here will give valuable information for a better interpretation of MRX measurements.

\section{Appendix A: Equation of motion for Brownian rotation}

Here, we briefly present a derivation of Eq. (4) for the Brownian rotation of the cluster. We employ the so-called "egg-model" used by Shliomis and Stepanov ${ }^{38}$ to treat the combined Néel and Brownian rotation of single-core magnetic particles. Define the rotational operators $\mathcal{L}_{\mathbf{e}_{i}}=\mathbf{e}_{i} \times \partial / \partial \mathbf{e}_{i}$ and $\mathcal{L}_{\mathbf{u}_{i}}=\mathbf{u}_{i} \times \partial / \partial \mathbf{u}_{i}$. Following Ref. 38, the torque balance for the magnetic moment $\mathbf{e}_{i}$ can be written as

$$
-\xi_{\mathrm{m}}\left(\boldsymbol{\omega}_{i}^{\mathrm{r}}-\boldsymbol{\omega}\right)-\mathcal{L}_{\mathbf{e}_{i}} \mathcal{H}=0
$$

where $\xi_{\mathrm{m}}$ is a magnetic friction coefficient and $\boldsymbol{\omega}_{i}^{\mathrm{r}}$ the relaxational part of the angular velocity of moment $\mathbf{e}_{i}$. Similarly, the torque balance for the particle orientation $\mathbf{u}_{i}$ reads ${ }^{38}$

$$
-\zeta_{1} \boldsymbol{\omega}-\xi_{\mathrm{m}}\left(\boldsymbol{\omega}-\boldsymbol{\omega}_{i}^{\mathrm{r}}\right)-\mathcal{L}_{\mathbf{u}_{i}} \mathcal{H}=0
$$

where $\zeta_{1}$ denotes the viscous friction coefficient of a single particle. The second term in Eq. (A2) denotes a braking torque dictated by Newton's third law. Adding Eqs. (A1) and (A2) and summing over all particles in the cluster leads to

$$
-N \zeta_{1} \boldsymbol{\omega}-\sum_{i=1}^{N}\left(\mathcal{L}_{\mathbf{e}_{i}}+\mathcal{L}_{\mathbf{u}_{i}}\right) \mathcal{H}=0
$$

Note that magnetic anisotropy does not contribute to $\left(\mathcal{L}_{\mathbf{e}_{i}}+\mathcal{L}_{\mathbf{u}_{i}}\right) \mathcal{H}$. Furthermore, due to the summation over all particles, also the total torque due to dipolar interactions vanishes. Therefore, we are left with the torque balance of the total viscous and total magnetic torque,

$$
-N \zeta_{1} \boldsymbol{\omega}+\sum_{i=1}^{N} m \mathbf{e}_{i} \times \mathbf{H}_{0}=0 .
$$

To arrive at Eq. (4), we replaced the friction coefficient $N \zeta_{1}$ with the friction coefficient of the cluster $\zeta_{N}$ and added the fluctuating torque $\mathbf{R}$.

\section{Appendix B: Numerical implementation}

We use the internal diffusion time $\tau_{0}=m /\left[2 \gamma \lambda k_{\mathrm{B}} T\right]$ to define the dimensionless time $t^{*}=t / \tau_{0}$ and write the LLG equation (1) in dimensionless form ${ }^{32}$

$$
\frac{\mathrm{d}}{\mathrm{d} t^{*}} \mathbf{e}_{i}=-\frac{1}{2 \lambda} \mathbf{e}_{i} \times\left(\mathbf{H}_{i}^{\mathrm{tot}}\right)^{*}-\frac{1}{2} \mathbf{e}_{i} \times\left(\mathbf{e}_{i} \times\left(\mathbf{H}_{i}^{\mathrm{tot}}\right)^{*}\right)
$$

with $\left(\mathbf{H}_{i}^{\text {tot }}\right)^{*}=\frac{m}{k_{\mathrm{B}} T} \mathbf{H}_{i}^{\text {tot }}$ and $\mathbf{H}_{i}^{\text {tot }}=\mathbf{H}_{i}+\mathbf{H}_{i}^{\mathrm{f}}$.

Rewriting the Stratonovich stochastic differential equation (B1) in the Itô interpretation, the corresponding EulerMaruyama integration scheme in the absence of Brownian rotation reads ${ }^{32}$

$$
\mathbf{e}_{i}(t) \rightarrow \mathbf{e}_{i}(t+\Delta t)=\frac{\mathbf{e}_{i}(t)+\Delta \mathbf{e}_{i}}{\left|\mathbf{e}_{i}(t)+\Delta \mathbf{e}_{i}\right|}
$$


with time step $\Delta t$ and

$$
\begin{aligned}
\Delta \mathbf{e}_{i}= & -\frac{\Delta t^{*}}{2}\left[\frac{1}{\lambda} \mathbf{e}_{i}(t) \times \mathbf{H}_{i}^{*}(t)+\mathbf{e}_{i}(t) \times\left(\mathbf{e}_{i}(t) \times \mathbf{H}_{i}^{*}(t)\right)-\mathbf{e}_{i}(t)\right] \\
& -\frac{1}{\sqrt{2\left(1+\lambda^{2}\right)}}\left[\mathbf{e}_{i}(t) \times \Delta \mathbf{W}_{t}^{*}+\lambda \mathbf{e}_{i}(t) \times\left(\mathbf{e}_{i}(t) \times \Delta \mathbf{W}_{t}^{*}\right)\right]
\end{aligned}
$$

where $\Delta t^{*}=\Delta t / \tau_{0}$ and $\Delta \mathbf{W}_{t}^{*}=\Delta \mathbf{W}_{t} / \sqrt{\tau_{0}}$ are independent increments of a three-dimensional Wiener process with variance $\Delta t^{*}$. For the corresponding Heun algorithm the reader is referred to Ref. 32 .

As a test of the algorithm, we consider the case $Q_{\mathrm{dd}}=0$ and $\mathbf{u}_{i}$ parallel to the $z$-axis, i.e. independent magnetic particles with identical orientation of their easy axis and no Brownian rotation. Apply an external magnetic field $\mathbf{H}_{0}$ that forms the angle $\chi$ with respect to the easy axis. In Fig. B we show the convergence of the average magnetic moment in field direction to the analytical value for decreasing time step. In agreement with earlier findings ${ }^{33}$, we observe that the Heun algorithm converges faster but that the Euler algorithm gives accurate results for $\Delta t^{*} \lesssim 0.005$. Since the Heun algorithm is computationally more expensive for the interacting system, we use in the following the Euler scheme with time step $\Delta t^{*}=0.0025$.

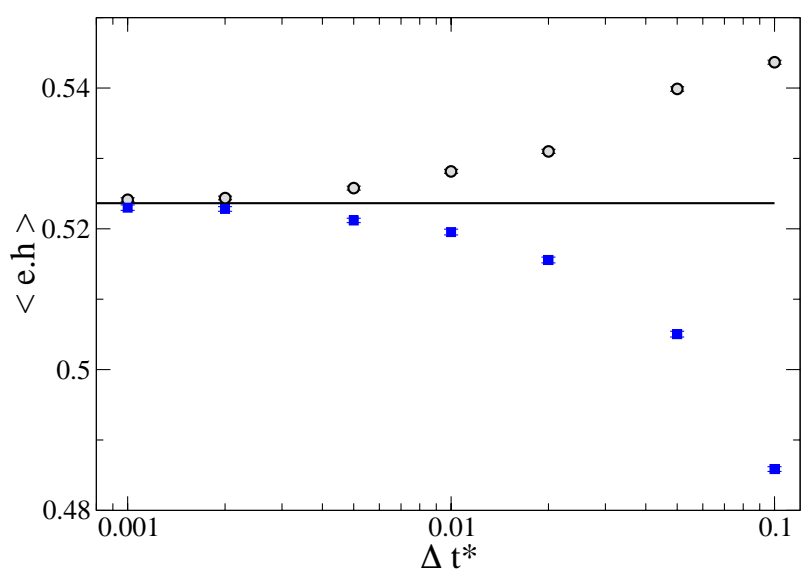

FIG. 10. Equilibrium magnetization parallel to applied field $\left\langle\mathbf{e}_{i} \cdot \mathbf{h}\right\rangle$ as a function of integration step size $\Delta t^{*}$. Easy axis and applied field form angle $\chi=2 \pi / 3$. Squares and circles denote the result of Euler and Heun algorithm, respectively. Horizontal line is analytical result. Other parameters are $\alpha=2$ and $\kappa=1$.

To simulate Brownian rotation, we also use an Euler-Maruyama scheme,

$$
\mathbf{u}_{i}(t) \rightarrow \mathbf{u}_{i}(t+\Delta t)=\frac{\mathbf{u}_{i}(t)+\Delta \mathbf{u}_{i}}{\left|\mathbf{u}_{i}(t)+\Delta \mathbf{u}_{i}\right|}
$$

where

$$
\begin{aligned}
\Delta \mathbf{u}_{i}= & \Delta t^{*}\left[\frac{1}{2} q \alpha\left(\mathbf{h}\left(\mathbf{M}^{*} \cdot \mathbf{u}_{i}(t)\right)-\mathbf{M}^{*}\left(\mathbf{h} \cdot \mathbf{u}_{i}(t)\right)\right)-q \mathbf{u}_{i}(t)\right] \\
& -\sqrt{q} \mathbf{u}_{i}(t) \times \Delta \mathbf{W}_{t}^{*}
\end{aligned}
$$

with $q=\tau_{0} / \tau_{\mathrm{B}}$ the ratio of the internal diffusion and the Brownian relaxation time and $\mathbf{M}^{*}=\mathbf{M}(t) / m=\sum_{j=1}^{N} \mathbf{e}_{j}(t)$.

To simulate the combined LLG and Brownian dynamics, we adapt the algorithm proposed in Ref. 33 to the present case:

1. Calculate the orientation of the magnetic moment and easy axis of the next time step separately: Brownian rotation:

$$
\begin{aligned}
& \mathbf{u}_{i}(t) \rightarrow \mathbf{u}_{i}(t+\Delta t)=\mathbf{u}_{i}(t)+\Delta \mathbf{u}_{i} \\
& \mathbf{e}_{i}(t) \rightarrow \mathbf{e}_{i}^{\mathrm{B}}(t+\Delta t)=\mathbf{e}_{i}(t)+\Delta \mathbf{u}_{i}
\end{aligned}
$$


Néel rotation

$$
\mathbf{e}_{i}(t) \rightarrow \mathbf{e}_{i}^{\mathrm{N}}(t+\Delta t)=\mathbf{e}_{i}(t)+\Delta \mathbf{e}_{i}
$$

2. Combine magnetic moments

$$
\mathbf{e}_{i}^{\prime}(t+\Delta t)=\mathbf{e}_{i}^{\mathrm{B}}(t+\Delta t)+\mathbf{e}_{i}^{\mathrm{N}}(t+\Delta t)-\mathbf{e}_{i}(t)
$$

3. Normalization

$$
\mathbf{e}_{i}(t+\Delta t)=\frac{\mathbf{e}_{i}^{\prime}(t+\Delta t)}{\left|\mathbf{e}_{i}^{\prime}(t+\Delta t)\right|}
$$

4. Rigid rotation of cluster

$$
\mathbf{r}_{i}(t+\Delta t)=\mathbf{r}_{i}(t)+\Delta \boldsymbol{\omega}^{*} \times \mathbf{r}_{i}(t)
$$

where $\Delta \boldsymbol{\omega}^{*}=\frac{1}{2} q \alpha \sum_{i} \mathbf{e}_{i} \times \mathbf{h} \Delta t^{*}+\sqrt{q} \Delta \mathbf{W}_{t}^{*}$.

\section{Appendix C: Relaxation}

As a further test of the algorithm, consider the relaxation dynamics of the average magnetization $\left\langle\mathbf{e}_{i}\right\rangle(t)$ from a perfectly oriented initial state $\mathbf{e}_{i}(0)=\mathbf{u}=(0,0,1)^{T}$ in the absence of an external field $\mathbf{H}_{0}$. We also disregard dipolar interactions $\left(Q_{\mathrm{dd}}=0\right)$ and neglect Brownian rotation. Therefore, we here study the magnetization relaxation of an individual magnetic moment due to the LLG alone. Figure $\mathrm{C}$ shows the resulting magnetization decay which follows nicely an exponential law. We fit the relaxation dynamics to $\left\langle e_{z}\right\rangle(t)=\exp [-t / \tau]$ and thereby extract the resulting relaxation time $\tau$. Results for effective relaxation time $\tau$ are shown in Fig. C. For large enough values of the anisotropy parameter $\kappa$, the relaxation time roughly follows Brown's result for high barriers, $\tau_{\mathrm{N}}=\tau_{0} \sqrt{\pi} e^{\kappa} /\left(2 \kappa^{3 / 2}\right)^{16}$.

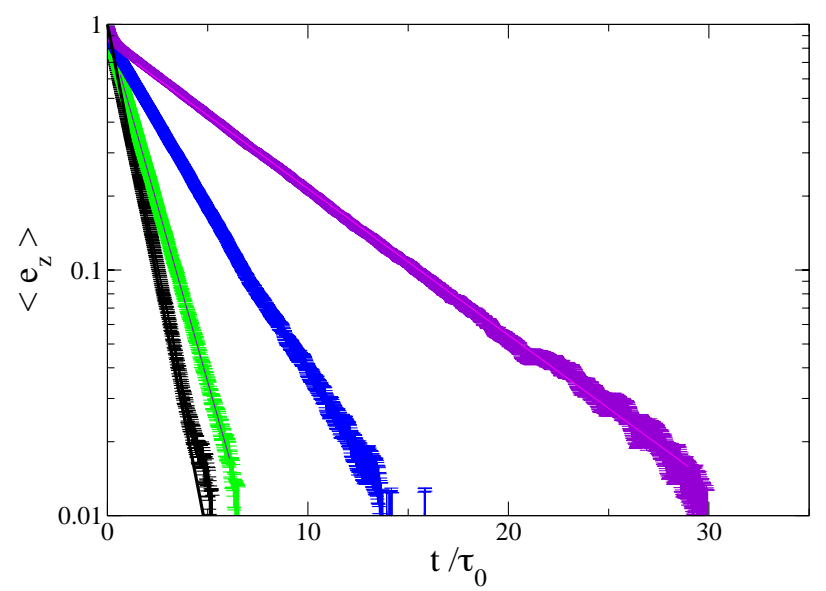

FIG. 11. Relaxation of mean orientation of magnetic moment $\langle\mathbf{e}\rangle(t)$ from a perfectly oriented state for anisotropy parameter $\kappa=0.5,1,2.5,4$ from left to right. The Euler algorithm with dimensionless time step $\Delta t^{*}=0.002$ was employed and damping parameter $\lambda=0.2$. Solid lines are exponential fits $\left\langle e_{z}\right\rangle(t)=\exp [-t / \tau]$. 


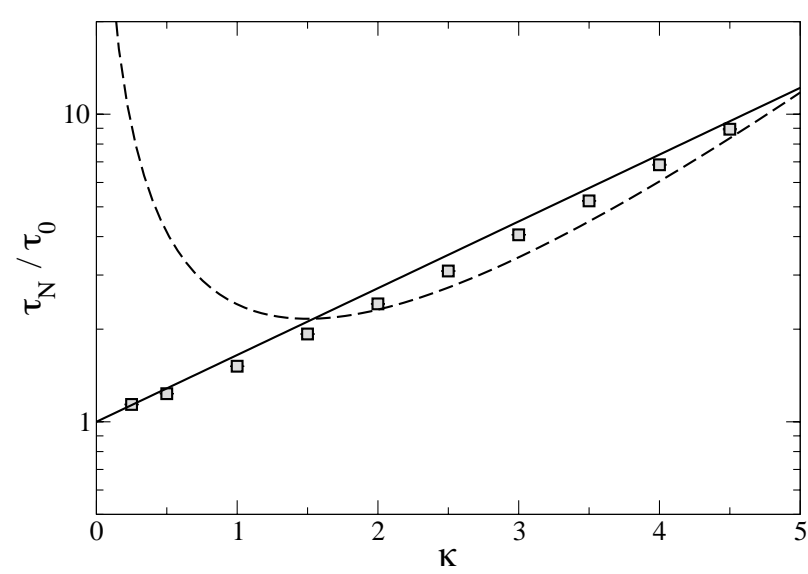

FIG. 12. Effective relaxation time $\tau_{\mathrm{N}} / \tau_{0}$ from relaxation $\langle\mathbf{e}\rangle(t)$ from perfectly oriented state. Dashed line is Brown's result for high barriers, $\tau_{\mathrm{N}}=\tau_{0} \sqrt{\pi} e^{\kappa} /\left(2 \kappa^{3 / 2}\right)$. Solid line corresponds to $\tau_{\mathrm{N}}=\tau_{0} \exp [\kappa / 2]$.

\section{ACKNOWLEDGMENTS}

Valuable discussions with Dietmar Eberbeck and Stefan Wenk are gratefully acknowledged. PI acknowledges support from the German Science Foundation (DFG) within the priority program SPP1681 "Field controlled particle matrix interactions: synthesis multiscale modelling and application of magnetic hybrid materials" under grant no. IL 122/1-1 as well as an EU FP7-MC-CIG Grant No. 631233.

1 A. Auge, A. Weddemann, F. Wittbracht, and A. Hütten, Applied Physics Letters 94, 183507 (2009).

2 A. Weddemann, F. Wittbracht, A. Auge, and A. Hütten, Applied Physics Letters 94, 173501 (2009).

3 M. Naous, D. García-Gómez, F. J. López-Jiménez, F. Bouanani, M. L. Lunar, and S. Rubio, Analytical Chemistry 89, 1353 (2016).

${ }^{4}$ M. Colombo, S. Carregal-Romero, M. F. Casula, L. Gutiérrez, M. P. Morales, I. B. Böhm, J. T. Heverhagen, D. Prosperi, and W. J. Parak, Chem. Soc. Rev. 41, 4306 (2012).

${ }^{5}$ Q. A. Pankhurst, N. T. K. Thanh, S. K. Jones, and J. Dobson, J. Phys. D: Appl. Phys. 42, 224001 (2009).

${ }^{6}$ R. Hergt, S. Dutz, R. Müller, and M. Zeisberger, J. Phys.: Condens. Matter 18, S2919 (2006).

7 S. Dutz, M. Kettering, I. Hilger, R. Müller, and M. Zeisberger, Nanotechnology 22, 265102 (2011).

8 C. Blanco-Andujar, D. Ortega, P. Southern, Q. A. Pankhurst, and N. T. K. Thanh, Nanoscale 7, 1768 (2015).

9 T. J. Yoon, H. Lee, H. Shao, S. A. Hilderbrand, and R. Weissleder, Advanced Materials 23, 4793 (2011).

10 D. Eberbeck, V. Janke, S. Hartwig, U. Heyen, D. Schüler, M. Albrecht, and L. Trahms, J. Magn. Magn. Mater. 289, 70 (2005).

11 F. Wiekhorst, C. Seliger, R. Jurgons, U. Steinhoff, D. Eberbeck, L. Trahms, and C. Alexiou, J Nanosci Nanotechnol 6, 3222 (2006).

12 F. Wiekhorst, U. Steinhoff, D. Eberbeck, and L. Trahms, Pharmaceutical research 29, 1189 (2012).

13 D. Eberbeck, C. Bergemann, S. Hartwig, U. Steinhoff, and L. Trahms, Journal Of Magnetism And Magnetic Materials 289, 435 (2005).

14 D. Soukup, S. Moise, E. Céspedes, J. Dobson, and N. D. Telling, Acs Nano 9, 231 (2015).

15 D. Eberbeck, M. Kettering, C. Bergemann, P. Zirpel, I. Hilger, and L. Trahms, Journal of Physics D Applied Physics 43, $405002(2010)$.

16 W. T. Coffey, P. J. Cregg, and Y. P. Kalmykov, in Advances in Chemical Physics, edited by I. Prigogine and S. A. RICE (1993), pp. 263-464.

17 E. Blums, A. Cebers, and M. M. Maiorov, Magnetic Fluids (de Gruyter, Berlin, 1997).

18 P. Ilg and M. Kröger, Phys. Rev. E 66, 021501 (2002), erratum: Phys. Rev. E, 67:049901, 2003.

19 P. Ilg and S. Odenbach, in Colloidal Magnetic Fluids: Basics, Development and Applications of Ferrofluids, edited by S. Odenbach (Springer, Berlin, 2008), vol. 763 of Lecture Notes in Phys. 
20 B. Fischer, J. Wagner, M. Schmitt, V. Trieu, and R. Hempelmann, Z. Phys. Chem. 220, 69 (2006).

21 D. V. Berkov, N. L. Gorn, and D. Stock, J. Magn. Magn. Mater. 310, e1015 (2007).

${ }^{22}$ F. Ludwig, O. Kazakova, L. F. Barquín, A. Fornara, L. Trahms, U. Steinhoff, P. Svedlindh, E. Wetterskog, Q. A. Pankhurst, P. Southern, et al., IEEE Transactions on Magnetics 50, 1 (2014).

${ }^{23}$ F. Ahrentorp, A. Astalan, J. Blomgren, C. Jonasson, E. Wetterskog, P. Svedlindh, A. Lak, F. Ludwig, L. J. Van IJzendoorn, F. Westphal, et al., J. Magn. Magn. Mater. 380, 221 (2015).

24 S. Dutz, J. H. Clement, D. Eberbeck, T. Gelbrich, R. Hergt, R. Müller, J. Wotschadlo, and M. Zeisberger, Journal Of Magnetism And Magnetic Materials 321, 1501 (2009).

25 D. Eberbeck, F. Wiekhorst, U. Steinhoff, and L. Trahms, Journal Of Physics-Condensed Matter 18, S2829 (2006).

${ }^{26}$ V. Schaller, G. Wahnström, A. Sanz-Velasco, P. Enoksson, and C. Johansson, Journal Of Magnetism And Magnetic Materials 321, 1400 (2009).

27 V. Schaller, G. Wahnström, A. Sanz-Velasco, S. Gustafsson, E. Olsson, P. Enoksson, and C. Johansson, Physical Review B 80, 092406 (2009).

${ }^{28}$ M. Woińska, J. Szczytko, A. Majhofer, J. Gosk, K. Dziatkowski, and A. Twardowski, Physical Review B 88, 144421 (2013).

${ }^{29}$ L. Lartigue, P. Hugounenq, D. Alloyeau, S. P. Clarke, M. Lévy, J.-C. Bacri, R. Bazzi, D. F. Brougham, C. Wilhelm, and F. Gazeau, Acs Nano 6, 10935 (2012).

30 M. F. Hansen, C. B. Koch, and S. Morup, Physical Review B 62, 1124 (2000).

31 A. Weddemann, A. Auge, D. Kappe, F. Wittbracht, and A. Hütten, Journal Of Magnetism And Magnetic Materials 322, $643(2010)$.

32 J. L. García-Palacios and F. J. Lázaro, Physical Review B 58, 14937 (1998).

${ }^{33}$ H. Rogge, M. Erbe, T. M. Buzug, and K. Lüdtke-Buzug, Biomedizinische Technik/Biomedical Engineering 58, 1 (2013).

34 J. L. Garcia-Palacios, On the statics and dynamics of magneto-anisotropic nanoparticles (John Wiley \& Sons, 2000).

35 S. I. Denisov, T. V. Lyutyy, and K. N. Trohidou, Physical Review B 67, 014411 (2003).

36 D. V. Berkov and N. L. Gorn, J. Phys.: Condens. Matter 14, L281 (2002).

37 J. K. G. Dhont, An introduction to dynamics of colloids, Studies in interface science (Elsevier, Amsterdam, 1996).

38 M. Shliomis and V. Stepanov, Journal Of Magnetism And Magnetic Materials 122, 196 (1993).

39 D. V. Berkov, N. L. Gorn, and D. Stock, J. Magn. Magn. Mater. 272, E1281 (2004).

40 M. Y. Lin, H. M. Lindsay, D. A. Weitz, R. KLEIN, R. C. Ball, and P. Meakin, Journal Of Physics-Condensed Matter 2, 3093 (1990).

41 P. Meakin, Physical Review B 28, 5221 (1983).

42 A. D. Melas, L. Isella, A. G. Konstandopoulos, and Y. Drossinos, Journal of Colloid and Interface Science 417, 27 (2014).

43 J. G. Kirkwood and J. Riseman, J. Chem. Phys. 16, 565 (1948).

44 J. A. Aronovitz and D. R. Nelson, Journal de physique 47, 1445 (1986).

45 D. X. Chen, A. Sanchez, E. Taboada, A. Roig, N. Sun, and H. C. Gu, Journal Of Applied Physics 105 (2009).

46 D. K. Lottis, R. M. White, and E. D. Dahlberg, Physical Review Letters 67, 362 (1991). 